\title{
Renin-angiotensin-aldosterone system blockade with telmisartan prevents the decline in cardiac contractility orchiectomy-dependent in SHR: role of PPAR-Y activation
}

\section{Marito Afonso Sousa Costa Silva}

Faculdade Multivix

\section{Camila Almenara}

UFOB: Universidade Federal do Oeste da Bahia

\section{Antonio Marcos Birocale}

UFES: Universidade Federal do Espirito Santo

\section{Renata Andrade Ávila}

FAESA: Faculdades Integradas Espirito-Santenses

Ivanita Stefanon

UFES: Universidade Federal do Espirito Santo

\section{Nazaré S Bissoli}

UFES: Universidade Federal do Espirito Santo

Alessandra Padilha ( $\nabla$ ale_padilha@hotmail.com )

UFES: Universidade Federal do Espirito Santo https://orcid.org/0000-0002-9585-1347

\section{Research Article}

Keywords: Testosterone, Myocardial contractility, Renin angiotensin system, PPAR Gama, papillary muscles

Posted Date: March 30th, 2021

DOl: https://doi.org/10.21203/rs.3.rs-332022/v1

License: (c) (1) This work is licensed under a Creative Commons Attribution 4.0 International License. Read Full License 


\section{Abstract}

The present study aimed to investigate the beneficial role of AT1 receptor blockade and PPAR-y activation in preventing cardiac contractile changes in hypertensive animals (SHR) with testosterone deficiency. Rats were divided into a control group (sham), orchiectomized (OCT), orchiectomized that received telmisartan (OCT + Tel) or telmisartan plus PPAR-y antagonist (OCT + Tel + BADGE). After 8 weeks, contractility of isolated papillary muscles was evaluated. The OCT group showed a reduction in the contraction force, avoided in the OCT + Tel and Tel + BADGE groups. Post-pause potentiation (PPP) was similar in the Sham, OCT and OCT + Tel group, suggesting prevention of sarcoplasmic reticulum activity. In contrast, PPP in the OCT + Tel + BADGE group decreased. The reduction in response to calcium or isoproterenol in the OCT group was prevented in the OCT + Tel and Tel + BADGE group. The influx of calcium, assessed indirectly, although it was similar in the OCT and Sham groups, increased both in the $\mathrm{OCT}+$ Tel groups and in the OCT + Tel + BADGE groups. SERCA2a protein expression was increased in the OCT group, which was avoided in the OCT + Tel and Tel + BADGE group. NCX expression was increased in the Tel + BADGE group compared to OCT + Tel and sham. Our results suggest that AT1 receptor blockade with telmisartan prevents compromised papillary muscle contractility in SHR. Moreover, it has been shown that the activation of PPAR-y adds benefits to the inhibition of renin-angiotensin system with telmisartan in preventing harmful effects on the contractility of hypertensive rats with testosterone deficiency.

\section{Introduction}

Steroid hormones, including testosterone, act on the cardiovascular system by promoting various membrane changes and modulating cell signaling [1]. Testosterone's ability to modulate cardiac function and blood pressure is already established by its action on the renin-angiotensin system (RAS) [2]. RAS is a complex system, well known for being involved in maintaining hemodynamic stability by regulating the electrolyte balance and blood pressure [3].

The development of hypertension is complex and is associated with the alteration of several systems. However, evidence suggests that the activation of the RAS is one of the protagonists of the genesis and maintenance of essential arterial hypertension [4-7] with implications for the structural and functional remodeling of the heart [8-10]. Angiotensin II (AngII) increases protein synthesis and collagen production via activation of (MAP) and kinase nd TGF- $\beta$, respectively, promoting myocardial hypertrophy and interstitial fibrosis $[11,12]$.

Epidemiological and clinical studies have shown that sex plays a determining role in the pathophysiology of hypertension and cardiac hypertrophy $[13,14]$. The mechanisms responsible for gender differences in pressure control are not yet well understood, however it has been shown that androgens, such as testosterone, play an important role in gender-related differences in blood pressure regulation $[15,16]$. Testosterone deficiency is associated with changes in cardiac contractility and these changes are related 
to changes in the actions of the RAS. Orchiectomy causes increased angiotensin-converting enzyme (ACE) activity and morphological and lusitropic changes in SHR animals [17].

Hypertension is a cardiovascular disease (CVD) with a high mortality rate and worldwide incidence and aging, especially in men, there is an increased likelihood of the onset of CVD, especially hypertension. Antagonists of this system have been used to treat hypertension and, among them, telmisartan, an AT1R antagonist that also has the ability to activate PPAR-y [18]. PPARs are a family of nuclear receptors known to regulate genes that participate in the metabolism of lipids, adipocytes and inflammation. PPARs are a family of nuclear receptors known to regulate genes that participate in the metabolism of lipids, adipocytes and inflammation. PPAR-y activation acts by decreasing oxidative stress and inflammation via protein-1 activation, the transducer transcriptional signal activator and the NF-KB pathway [19]. Moreover the use of PPAR-y agonists appears to be a new mechanism to mediate the beneficial effects of Angio II receptor antagonists [20,21]. Based on this, the hypothesis is that telmisartan, as an AT1 blocker and partial PPAR agonist, could reverse the compromised contractility of the papillary muscles isolated from orchiectomized SHR.

Therefore, this study aims to investigate if the block de AT1 receptor and activation de the PPAR-Y prevent these contractile changes in hypertensive animals with testosterone deprivation.

\section{Matherial And Methods}

EXPERIMENTAL ANIMALS. The use and care of these experimental animals followed the ethical principles of animal research, established by the Brazilian College of Animal Experimentation (COBEA1991). The Research Ethics Committee on Experimentation and Use of Animals at UFES (CEUA-UFES 064/2012) approved all experimental protocols. This study used spontaneously hypertensive rats (SHR) of three months of age obtained in the central vivarium of the Post-Graduate Program in Physiological Sciences at the Federal University of Espírito Santo (UFES). They were kept in cages under controlled temperature conditions and a 12-hour light-dark cycle. The rats had free access to water and food.

ORCHIECTOMY. For orchiectomy surgery, the SHR were anesthetized and an anterior median cut was made in the scrotum, allowing the visualization of each testicle. The vas deferens was carefully isolated, connected and ruptured, allowing removal of the testis. The incision was then closed and sutured with 3 - 0 chromium catgut.

EXPERIMENTAL MODEL. The animals were divided into five groups: a control group (Sham), and the orchiectomized group (OCT) that received the vehicle ( $0.5 \%$ sodium carboxymethyl cellulose; $\mathrm{CMC}-\mathrm{Na}$ ) and an orchiectomized group treated with telmisartan daily by gavage ( $5 \mathrm{mg} / \mathrm{kg} /$ day for 8 weeks).

To investigate the effects of telmisartan on arterial blood pressure and the non-specific activation of PPAR- $y$, other groups of orchiectomized SHR received hydralazine (OCT + Hidra) via gavage (10 $\mathrm{mg} / \mathrm{kg} /$ day) or telmisartan plus the PPAR-y antagonist, BADGE (OCT + Tel + BADGE, $30 \mathrm{mg} / \mathrm{kg} /$ day i.p.). 
PONDERAL PARAMETERS. All groups were weighed at the beginning and end of the treatments to evaluate whether orchiectomy and telmisartan treatment had influence on weight gain. The animals were sacrificed after eight weeks, and their hearts were separated, weighed and corrected by the tibia length.

THE SYSTOLIC ARTERIAL BLOOD PRESSURE MEASUREMENTS. In order to eliminate the depressive effect of anesthesia, pressure was measured in an indirect and non-invasive manner at the beginning and at the end of the orchiectomy and at the end of all treatments. For this, the systolic arterial pressure (SAP) of rats of all groups was measured using the tail cuff plethysmography method (non-invasive blood pressure IITC Life Science, version 1.35). The rats of the five groups, previously acclimated, were placed in a heated chamber $\left(37^{\circ} \mathrm{C}\right)$ for 5 to 10 minutes to perform measurements through inflation-deflation cycles. Next, SAP was measured and the average of three measurements was recorded and analyzed.

\section{MYOCARDIAL CONTRACTILITY EVALUATION IN THE PAPILLARY MUSCLE.}

After 8 weeks of treatment, the rats were anesthetized with intraperitoneal urethane injection $(1.2 \mathrm{~g} / \mathrm{kg})$. The hearts were then rapidly removed and the posterior papillary muscle was fixed by rings, and attached at one fixed end and another attached to a force transducer (TSD125 - Byopac Systems, Inc; CA) coupled to an amplifier (DA100C Byopac Systems, Inc; CA), inside glass chambers filled with $20 \mathrm{~mL}$ of and perfused with Krebs-Henseleit solution in $\mathrm{mM}: \mathrm{NaCl} 120 ; \mathrm{KCl}$ 5.4; $\mathrm{CaCl} 21.25 ; \mathrm{MgCl} 2$ 1.2; $\mathrm{NaH} 2 \mathrm{PO} 42$; $\mathrm{Na} 2 \mathrm{SO} 4$ 1.2; $\mathrm{NaHCO} 18$ and glucose 11. pH was kept 7.4 using $95 \% \mathrm{O} 2$ and $5 \% \mathrm{CO} 2$ ) at $29^{\circ} \mathrm{C}$ to avoid the possibility of hypoxia [22]. The papillary muscles were electrically stimulated using a pair of platinum electrodes (rectangular pulses, duration $12 \mathrm{~ms}$ and voltage 1.5 times the threshold, approximately $10 \mathrm{mV}$, $0.5 \mathrm{~Hz}$ ). Muscles were stretched until reaching the maximum active tension muscle length ( $\mathrm{Lmax}$ ) and the experimental protocols began after stabilizing the preparations for 60 minutes. The developed force was recorded in a data acquisition system (MP100 Byopac Systems, Inc; CA), at 500 samples/second sampling rate. At the end of the experiment, the papillary muscles were weighed, and the isometric contraction force developed was adjusted by the weight of the muscles.

After the stabilization period, the isometric contraction: force expressed in grams per milligram of muscle weight $(F ; g / m g)$, the activation times (times from the beginning of the contraction to the maximum peak of strength) and relaxation (maximum peak time up to $50 \%$ of isometric relaxation) and those derived from positive and negative force time ( $\mathrm{dF} / \mathrm{dt} ; \mathrm{g} / \mathrm{mg} / \mathrm{s})$ were evaluated in all experimental groups [23].

Relative post-pause potentiation (PPP \%) was performed to indirectly obtain inferences on the functional activity of the mechanisms of calcium uptake (sarcoplasmic reticulum-SR and $\mathrm{Na}^{+} / \mathrm{Ca}^{2+}$ exchangerNCX). The potentiation was obtained by electrical re-stimulation after 15-, 30- and 60-second pauses in the electrical stimulus applied to the muscle [24]. Relative potentiation was considered as the ratio between contraction amplitude after the pause, and the contraction amplitude before the pause, aiming to evaluate the SR activity $[25,26]$ in papillary muscles of the control and untreated groups.

To evaluate the inotropic response to calcium, a calcium concentration-response curve to extracellular calcium was performed using $0.62,1.25,2.5$ and $3.75 \mathrm{mM} \mathrm{CaCl}_{2}$ in the perfusion solution. The force 
variation was corrected by the weight of the muscles and expressed in $\mathrm{g} / \mathrm{mg}$.

Contractions obtained after a 10-minute pause in electrical stimulation (PRC) were performed to evaluate the interference of orchiectomy and telmisartan treatment on the inflow of trans-sarcolemmal calcium.

For this, the calcium-free $\mathrm{KH}$ solution and $10 \mathrm{mM}$ Caffeine were used for depletion of intracellular calcium and SR content. The preparations were re-perfused with normal $\mathrm{KH}$ solution seconds before restarting the stimulation procedures. From this method, it can be inferred that the contraction after the 10-minute pause depends on the calcium coming from the extracellular medium, since the intracellular stores were depleted.

To assess whether orchiectomy and telmisartan treatment was able to alter $\beta$-adrenergic responsiveness, a concentration-response curve to isoproterenol $\left(10^{-11}\right.$ to $\left.10^{-5} \mu \mathrm{M}\right)$ was performed.

\section{WESTERN BLOT ANALYSIS}

Frost samples of the heart were grinded with ice-cold RIPA buffer (Sigma Aldrich) using a mixer. The lysate was centrifuged at 6,000 RPM, the supernatant was collected, and the protein concentration was determined by Bradford assay (Bio-rad). Aliquots containing $40 \mathrm{ug}$ of protein were made for each animal and loaded into $7.5,10$ or $12 \%$ acrylamide TGX Stain-Free gels (Bio-rad). The protein collection of the samples was separated by electrophoresis in a Mini-PROTEAN Tetracell system (Bio-rad) for $2 \mathrm{~h}$ at $120 \mathrm{~V}$ in a running buffer containing $140 \mathrm{mM}$ glycine, $37 \mathrm{mM}$ Tris-Base and 1\% Sodium Dodecyl Sulfate (Sigma Aldrich). The gels were then activated with UV irradiation (ChemiDocXRS + Image System, Bio-rad) for 1 minute, producing fluorescent signal by tryptophan residues present on the proteins. The protein content was wet transferred to a nitrocellulose membrane (Bio-rad) for $18 \mathrm{~h}$ at $0.25 \mathrm{~A}$ in an ice-cold transfer solution containing $140 \mathrm{mM}$ glycine, $37 \mathrm{mM}$ Tris-base and $20 \%$ of methanol. The total protein content for each sample was acquired by imaging the fluorescence emission (ChemiDoc XRS+, Bio-rad). The membranes were blocked with 5\% skimmed milk (Molico, Nestlé) in Tris-buffered solution with Tween 20 (TBST) for $1 \mathrm{~h}$ under agitation at room temperature, and incubated with the following primary antibodies: anti-NCX 1:1000 (Thermo Fisher), anti-SERCA2 ATPase 1:1000 (Thermo Fisher), anti-AT1 1:250 (Santa Cruz), anti- PPAR-y 1:500 (Santa Cruz), anti-Phospholamban 1:1000 (Thermo Fisher) or phosphorylated anti-Phospholamban ser16. The incubations underwent stirring overnight at $4{ }^{\circ} \mathrm{C}$. After thorough washing, the membranes were incubated with Strep Tactin HRP-conjugate 1:5000 and adequate secondary antibody: anti-mouse 1:5000 (Sigma Aldrich) or anti-rabbit 1:5000 (Sigma Aldrich); all membranes were placed in 3\% bovine serum albumin (Sigma) in TBST for $1 \mathrm{~h}$, under stirring, after being washed with TBTS. A chemiluminescent substrate $(0.2 \mathrm{mM}$ cumaric acid, $1.25 \mathrm{mM}$ luminol, $0.1 \mathrm{M}$ Tris$\mathrm{HCL}$ and $0.06 \%$ Hydrogen Peroxide) was applied to the membranes and pictures were taken with ChemiDoc XRS+. The intensity of luminescence was quantified and normalized by the total protein content of the sample using Image Lab 6.0.1 Software (Bio-rad).

\section{HISTOLOGICAL ANALYSES}


The left ventricle (LV) was sectioned in the middle, and the apical half of each ventricle was fixed in phosphate buffer solution with $4 \%$ formalin $(0.01 \mathrm{mM}, \mathrm{pH} 7.4)$. After the fixation period, the LV was placed in a plastic cassette of processor/inclusor type. The cassettes were processed in an automated apparatus with a 12-hour total cycle for dehydration in solutions with increasing concentrations of ethyl alcohol until reaching absolute (100\%), and diaphanization in xylol. The material was embedded in paraffin and cut into a $5 \mu \mathrm{M}$ thick Leica microtome. The sections were placed on a glass slide and exposed to a temperature of $37^{\circ} \mathrm{C}$ for drying. The material was dewaxed in xylol and hydrated in alcohol for Hematoxylin and Eosin staining for the analysis of cardiac hypertrophy, and picrosirius red staining for the analysis of collagen deposition.

After staining, the slides were visualized under a light microscope (LEICA DM/LS) coupled to a camera. Three photos were taken for each slide and the images were analyzed by $\mathrm{J}$ software. For evaluation of cardiac hypertrophy, the size of the nucleus - stained with the hematoxylin, eosin method - was estimated in images obtained at $400 \times$ magnification, considering only the fully visible nuclei. These presented an ellipsoid shape in manual selection. Horizontally or transversely cut nuclei were discarded. To evaluate collagen deposition in the myocardium, the analyses were conducted using a minimum limit of red at 87 , and a manually adjusted maximum at approximately 160 . Based on these values, the $\mathrm{J}$ software presented the percentage of the marked area corresponding to the collagen coloration by picrosirius red. Three visual fields of each sample were analyzed, and the mean of these values was calculated for the individual value of the sample and each group was composed of at least four samples.

\section{CHEMICALS}

The following drugs were used: anhydrous caffeine (B. Herzog, Brazil), urethane, bovine serum albumin, isoproterenol (Sigma Chemical Co., USA), telmisartan (micardis), hydralazine (Sigma Chemical Co., USA), sodium carboxymethylcellulose; CMC-Na (Sigma Chemical Co., USA) and PPAR-y inhibitor (Santa Cruz biotechnology). All other chemicals were of analytical reagent grade and were obtained from Sigma and Merck (Germany) or Reagen (Brazil).

\section{STATISTICAL ANALYSIS}

Results are expressed as mean \pm standard error of the mean (SEM). The values of " $n$ " represent the number of animals or samples used in each experimental protocol. Statistical analysis of the results was performed by two-way analysis of variance (ANOVA). When the analysis of variance was significant, it was followed by Fischer's post-hoc test. Statistical significance was considered when $P<0.05$. For the statistical analyses and the plotting of all graphs were made using GraphPad Prism 6.0 (San Diego, CA, USA).

\section{Results}

No differences in body weight were observed in Sham and OCT groups either before and after 8 weeks of treatment. However, telmisartan treatment or telmisartan plus BADGE reduced the weight of the OQT rats similarly (Table 1$)$. 
Table 1

Evaluation of body weight and blood pressure

\begin{tabular}{|c|c|c|c|c|}
\hline & $\begin{array}{l}\text { Corporal } \\
\text { mass }(g)\end{array}$ & $\begin{array}{l}\text { Heart/ tibia } \\
(\mathrm{g} / \mathrm{mm})\end{array}$ & $\begin{array}{l}\text { Systolic blood pressure } \\
(\mathrm{mmHg})\end{array}$ & $\begin{array}{l}\text { n } \\
\text { (group) }\end{array}$ \\
\hline Sham & $315.87 \pm 4.9$ & $35.35 \pm 1.9$ & $186.67 \pm 3.9$ & 9 \\
\hline ОСТ & $296.10 \pm 6.2$ & $31.77 \pm 1.6$ & $184.84 \pm 8.8$ & 11 \\
\hline $\begin{array}{l}\text { OCT + } \\
\text { hidralazine }\end{array}$ & $318 \pm 13.63$ & $45.73 \pm 3.2^{\star}$ & $111.97 \pm 1.9 * \#$ & 6 \\
\hline OCT + Tel & $282.90 \pm 6.5^{\star}$ & $27.24 \pm 2.4^{\star \#}$ & $109.34 \pm 1.6^{\star \#}$ & 13 \\
\hline $\begin{array}{l}\text { OCT + Tel + } \\
\text { BADGE }\end{array}$ & $278 \pm 10.6$ *\$ & 37.86×1.8@ & $106.48 \pm 1.3^{\star \#}$ & 9 \\
\hline
\end{tabular}

Cardiac hypertrophy was evaluated in all groups because of its relationship with an increase in the pressure overload, which is an adaptive response to pathological conditions such as hypertension (COOPER IV G, 1987). There was no significant difference in the heart weight/tibia length ratio between Sham and OQT groups (Table 1). However, telmisartan treatment reduced hypertrophy in hypertensive animals (Table 1). PPAR-y inhibitor impaired the anti-hypertrophic effect of telmisartan, suggesting that the activation of PPAR-y contributes to the improvement found in the OCT + Tel group (Table 1).

\section{BLOOD PRESSURE}

Systolic arterial pressure was evaluated at the beginning and at the end of the treatments by the tail-cuff plethysmography technique. As shown in Table 1, orchiectomy was not effective in changing SAP. However, as expected, the SAP in the OCT + Tel or OCT + Tel + BAGDE groups were reduced (Sham: 186.6 \pm 3.86; OCT: $184.8 \pm 8.7$; OCT + Tel: $109.9 \pm 1.61 *$; OCT + Tel + BADGE: $106.4 \pm 1.30 *$ mm Hg;)

\section{PAPILLARY MUSCLE CONTRACTILITY}

As shown in the Fig. 1A, 8-weeks after orchiectomy, there was a reduction in the contraction force of SHR isolated papillary muscles. However, the reduction of the contraction force in OCT group was prevented by telmisartan treatment (Fig. 1A). In addition, in the orchiectomized group treated with telmisartan plus BADGE the reduction of the contraction force, observed in OCT group, was restored at the same value observed in the Sham group (Fig. 1A).

To rule out the effect of arterial blood pressure reduction on our findings, another group was treated with hydralazine for 8 weeks (OCT + Hidra: $111.97 \pm 1.9 \mathrm{mmHg}$ ). As shown in the Fig. $1 \mathrm{~A}$, the treatment with hydralazine did not prevent the reduction of the contraction force of the papillary muscles at the end of the treatment. Therefore, data on the treatment with hydralazine are not shown for the other protocols. The positive force derivatives ( $\mathrm{dF} / \mathrm{dt}+$ ) were reduced in the orchiectomized group when compared to the 
Sham group (Fig. 1D), which was prevented by telmisartan treatment. In addition, the treatment with telmisartan plus BADGE improved the $\mathrm{dF} / \mathrm{dt}+$ when compared to Sham group (Fig. 1D). On the other hand, negative force derivatives ( $\mathrm{dF} / \mathrm{dt}-$ ) remained unchanged in all groups (Fig. 1E).

When evaluating the temporal parameters of contractility, the relaxation time remained unchanged (Fig. 1C) and the activation time showed a reduction only in the OCT + Tel + BADGE group when compared to the sham group and the OCT group (Fig. 1B).

In order to evaluate which mechanisms would be involved in reducing contraction induced by OCT, the first experimental protocol performed was the indirect evaluation of sarcoplasmic reticulum (SR) activity, as described in the methodology. Results suggest that SR activity was similar in Sham, OCT and OCT + Tel groups (Fig. 2). In contrast, OCT rats treated with telmisartan plus BADGE showed a decrease in the post-pause potentiation, suggesting a reduction of SR activity (Fig. 2).

Another important analysis of our study was to investigate whether orchiectomy and telmisartan treatment could modify the inotropic response of the isolated papillary muscles. For this, concentrationresponse curves to extracellular calcium and isoproterenol were performed (Fig. 3).

The inotropic response to extracellular calcium decreased in the OCT group when compared to the Sham group (Fig. 3A). However, telmisartan treatment prevented the reduction of the inotropic response to calcium in the OCT group (Fig. 3A). In addition, OCT rats, treated with telmisartan plus BADGE, showed an increased in the inotropic response to calcium when compared to other groups (Fig. 3A).

Similarly, to the inotropic response to calcium, isolated papillary muscle of OCT rats showed a reduction in the inotropic response to isoproterenol when compared to Sham rats (Fig. 3B). However, telmisartan treatment prevented the reduction of isoproterenol response in OCT rats (Fig. 3B). In addition, the treatment with telmisartan plus BADGE increased, even more, the response induced by isoproterenol when compared to the OCT + Tel group (Fig. 3B).

To assess whether the decrease in the inotropic response to calcium, observed in OCT rats, could be related to changes in calcium influx, CRP was performed as mentioned in the methodology. According to our results, PRC was similar in papillary muscles of OCT rats when compared to Sham rats. However, the treatment with telmisartan or telmisartan plus BADGE, increase this response in $39 \%$ and $110 \%$, (Sham:19.09 \pm 1.44; OCT:18.24 \pm 1.44; OCT + Tel:25.49 $\pm 1,2$; OCT + Tel + BADGE: $40,75 \pm 4,8$ force: $\mathrm{g} / \mathrm{mg}$ respectively, when compared to OCT group (Fig. 3C).

In order to clarify the functional results observed in papillary muscle, we analyzed the protein expression of SERCA 2A, NCX, PLB, PLB Ser16, AT1 and PPAR-y.

Orchiectomy increased SERCA 2A without altering significantly NCX, total PBL, PBL ser16 and AT1 proteins expression (Fig. 3D, E, F, G and H). However, telmisartan treatment prevented the increase of SERCA 2A and NCX protein expression was lower when compared to OCT rats, but reduced the PBL 
expression (Figs. 3D, E, and F). In addition, telmisartan treatment increased AT1 protein expression (Fig. 3H).

On the other hand, telmisartan plus BADGE treatment did not prevented the increase of NCX expression observed in OCT rats (Fig. 3E). However, similarly, that occurred with telmisartan treatment, telmisartan plus BADGE prevented the increase of SERCA 2A expression in OCT rats (Fig. 3). Furthermore, treatment with telmisartan and BADGE further reduced the expression of PLB when compared to treatment with telmisartan alone (Fig. 3F). Neither AT1 expression nor PPAR Gama expression were altered by telmisartan plus BADGE treatment (Figs. $3 \mathrm{H}$ and I).

\section{HISTOLOGICAL ANALYSES}

Although cardiac muscle mass did not change, the orchiectomized animals showed reduction in the perimeter (Fig. 4A) and nuclear area (Fig. 4B), and this effect was prevented by the inhibition of PPR-y, as shown in Fig. 4. This reduction is even more pronounced in the OCT + TEL group, indicating a joint role of RAAS in this process.

The reduction in nuclear size indicators was also not accompanied by changes in collagen content (Fig. 4C).

\section{Discussion}

According to the present data, treatment with telmisartan prevents damage to contractility orchiectomydependent, in papillary muscles isolated from SHR. Orchiectomized rats showed a reduction in the inotropic response to calcium and beta-adrenergic activation, without impairment of calcium influx and preserving arterial blood pressure.

Telmisartan treatment, on the other hand, seems to increase the transsarcolemal influx of calcium, which may be the main mechanism by which the drop in response to calcium and an adrenergic activation was prevented. This suggestive increase in calcium influx was accompanied by a reduction in protein expression of NCX and total phospholambam, which suggests greater intracellular calcium handling.

Several studies have already demonstrated that testosterone deprivation is able to decrease the left ventricular contractility [27-32]. Mechanisms that include increased relaxation time, reduced maximum myofilament response to calcium and the activity of ATPase Myosin, reduced maximum SERCA response [31] and decreased calcium content in SR [32] have already been described.

In the present study, the orchiectomized animals showed a reduction in isometric strength as a result of reduced response to calcium and beta-adrenergic activation. However, the speed of contraction and relaxation were not altered, as well as the function of RS, which suggests that there was preservation of the function of the calcium extrusion mechanisms, even in the condition of orchiectomy. 
Nevertheless, SERCA 2A protein expression was increased in the orchiectomized group (OCT) compared to the other groups, without changing the expression of NCX and PBL, which suggested that the calcium uptake by SR was not impaired. This is reflected in the potentiation result after pause and relaxation time, which are similar between the groups and indicate, albeit indirectly, that the calcium transit was not altered in this model of orchiectomy. Previous study demonstrated that orchiectomy in normotensive rats increases the sensitivity of SERCA 2A to calcium, but its maximum activity is reduced, without altering its protein expression, which reflected in the increase in the relaxation time (TC50\%) of hearts of orchiectomized rats [31]. In our orchiectomy model, we believe that the increase in SERCA 2A protein expression found may be a response to this change in the sensitivity and maximum activity of SERCA 2A, which may have culminated in the normalization of the relaxation time of the isolated papillary muscles.

Because our results suggest that apparently the SR function and the transarcolemal calcium influx have not been altered, we speculated that the orchidectomy induced a reduction in the sensitivity of contractile proteins. In fact, a previous study show that testosterone deficiency can alter the calcium interaction with contractile proteins, besides reducing the activity of myosinic ATPase and sensitivity of contractile proteins, which, in turn, could reducing cardiac contraction force in normotensive animals [31].

In addition, testosterone modulates cardiac function by affecting the renin-angiotensin system. As already shown, testosterone induces an increase in the production of angiotensinogen and increased renin activity resulting in an enhancing in angiotensin II production [5, 33]. Indeed, we observed that the inhibition of the renin-angiotensin system might improves the contractility of hypertensiveorchiectomized rats. Moreover, the treatment with telmisartan prevented the decline of inotropic responses induced by calcium and isoproterenol observed in OCT rats, probably by increasing of transarcolemal calcium influx. In addition, telmisartan treatment prevented the increased SERCA 2A protein expression observed in OCT group besides induces a reduction NCX and the total PBL protein expression in OCT rats.

These data suggest that the treatment with telmisartam, in addition to improving the calcium influx, may be increasing the calcium content in SR, due to the greater activity of SERCA. The reduction in NCX protein expression translates into less calcium extrusion by the sarcolemma and the reduction in PBL makes up a scenario where there will be a greater availability of myoplasmic calcium compared to a more efficient SERCA. Together, our results suggest an important relationship between testosterone deficiency and RAS, resulting in impairment of cardiac function. Furthermore, it also shows that the use of an RAS antagonist can bring benefits to the contractile dysfunction of the heart when an occurring association of hormonal deprivation and hypertension.

We observed no changes in AT1 protein expression in the OCT group. However, an increase of AT1 protein expression was observed after telmisartan treatment, probably due to positive feedback on prolonged inhibition of these receptors. It is possible to note that the AT1 protein expression was not altered in orchiectomized rats treated with telmisartan plus BADGE. This result suggests that the positive feedback observed in the OCT + Tel group probably depends on the activation of PPAR-Y. 
Telmisartan is an AT1 receptor antagonist with pharmacological properties defined by a long duration of action and high lipophilicity [20]. It also has partial agonistic properties of PPAR-y (a nuclear transcription factor receptor superfamily) that may confer beneficial properties in hypertensive patients [34]. It has been used in some studies to demonstrated the multiple changes caused by diseases such as hypertension, diabetes, myocardial infarction and coronary artery disease [35-38]. However, few studies show the effect of this drug on myocardial contractility, which further highlights the importance of our study. Telmisartan is used mainly due to its effect on RAS, but the effects on activate PPAR-y has been explored in some studies. According to these studies, PPAR- $\gamma$ activation appears to be an innovative mechanism to mediate the beneficial effects of inhibitors angiotensin receptors $[20,21,34,36]$.

To evaluate the participation of PPARy in preventing the fall of contractility observed in the telmisartan group, the OCT + Tel + BADGE group was added. Thus, we observed that the prevention of decline in contractility after treatment with telmisartan was maintained after treatment with telmisartan plus BADGE.

However, the inotropic responses induced by calcium and isoproterenol were increased in telmisartan plus BADGE group when compared to the group that received only telmisartan. This result suggests that the effect of telmisartan in preventing the impairment of contractility on OCT group is greater when occur select blocking AT1 receptor (Fig. 3).

Interestingly, when we indirectly assessed the calcium uptake by SR, using the PPP protocol, the telmisartan plus BADGE group showed a reduction compared to the other groups. For right, an increase of NCX protein expression and a reduction of total PLB without changing in SERCA protein expression was observed in the telmisartan plus BADGE group. Together, these results suggest that calcium is probably being extruded mainly by sarcolemma instead of being recaptured by $\mathrm{SR}$, which in turn could reduce the PPP in telmisartan plus BADGE group.

Calcium is one of the most abundant ions in the extracellular environment and its presence in large quantities in the intracellular environment can trigger several other deleterious mechanisms for the heart, such as activation of apoptotic pathways and SR saturation with the possibility of arrhythmia due to spontaneous emissions of SR calcium [39]. Thus, the upregulation of NCX observed in the telmisartan plus BADGE group may have been a compensatory mechanism for this intracellular calcium increase.

In this context, we suggest that a possible increase in calcium influx with the use of the PPAR y inhibitor. In addition to the increase in PRC, there was an increase in the responsiveness to calcium and betaadrenergic activation in telmisartan plus BADGE group when compared to the group treated only with telmisartan. Since the responsiveness to calcium involves the entry of calcium through the sarcolemma and the saturation of the calcium-binding sites in the sarcomere, the results obtained suggest that the effect of PPAR y inhibition occurs mainly by increasing the calcium inflow without changing the sensitivity of contractile proteins. 
It is known that sex hormones and RAS have the ability to induce changes in cardiac morphology. Cardiac hypertrophy is a mechanism in which cardiac mass is increased in response to functional overload that can influence myocardial contractile function. In our findings, orchiectomy did not change the heart's weight either the heart collagen content. However, the perimeter and the nuclear area were reduced, suggesting that testosterone deficiency seems to reduce cardiac trophism, without altering collagen deposition. This result also suggests that the impairment of cardiac force in OCT group does not involve morphological alteration.

In conclusion, it has been shown that orchiectomy causes impairment in the contractility of SHR papillary muscles. Likewise, it has been shown that this damage was prevented by treatment with telmisartan, probably by increasing the transarcolemal flow of calcium, with a consequent increase in the inotropic responses of the heart. In addition, this study created evidence that activation of PPAR-y may contribute to the beneficial effects of RAS inhibition in some diseases, such as hypertension.

\section{Declarations}

\section{FUNDING}

This study was supported by grants from Coordenação de Aperfeiçoamento de Pessoal de Nível Superior (CAPES - Finance code 001); Conselho Nacional de Desenvolvimento Científico e Tecnológico (CNPq Grant 303390/2015-8); and Fundação de Amparo a Pesquisa do Espírito Santo (FAPES - Grant $80600115 / 17)$. The funders had no role in study design, data collection and analysis, decision to publish, or preparation of the manuscript.

\section{COMPETING INTERESTS}

The authors state no conflict of interest.

\section{ETHICAL APPROVAL}

All procedures were approved and performed in accordance with the guidelines of the Research Ethics Committee on Experimentation and Use of Animals at UFES (CEUA-UFES 064/2012).

\section{AUTHOR CONTRIBUTIONS}

The authors declare that all data were generated in-house and that no paper mill was used. Silva MASC, Almenara C, Birocale AM and Ávila RA performed the experiments, analyzed the data and wrote the manuscript. Padilha AS, Stefanon I and Bissoli NS wrote the manuscript and designed the study.

\section{DATA AVAILABILITY}

The datasets generated during and/or analysed during the current study are available from the corresponding author on reasonable request. 


\section{References}

1. Golden KL, Marsh JD, Jiang Y, Moulden J (2004) Gonadectomy alters myosin heavy chain composition in isolated cardiac myocytes. Endocrine 24:137-140

2. Komukai K, Mochizuki S, Yoshimura M (2010) Gender and the renin-angiotensin-aldosterone system. Fundam Clin Pharmacol 24:687-698

3. FORRESTER SJ, BOOZ G W., SIGMUND C D., COFFMAN T M., KAWAI T (2018) RIZZO V, SCALIA R ES. Angiotensin II Signal Transduction. Angiotensin II Signal Transduct An Updat Mech Physiol Pathophysiol. Physiol Rev 98:1627-1738

4. Hall JE, Brands MJHJ (1999) Angiotensin II and long-term arterial pressure regulation: the overriding dominance of the kidney. J Am Soc Nephrol 10:S258-S265

5. Hall JE, Guyton ACBM. Control of sodium excretion and arterial pressure by intrarenal mechanisms and the renin-angiotensin system. In: Laragh JH, Brenner BM, eds. Hypertens Pathophysiol Diagnosis, Manag 2nd ed New York, NY Raven Press. 1995;1451-1475

6. James GD, Sealey JE, Müller F, Alderman M, Madhavan S, Laragh JH (1986) Renin relationship to sex, race and age in a normotensive population. J Hypertens Suppl 4:S387-S389

7. Rajagopalan S, Kurz S, Münzel T, Tarpey M, Freeman BA, Griendling KK et al (1996) Angiotensin IImediated hypertension in the rat increases vascular superoxide production via membrane $\mathrm{NADH} / \mathrm{NADPH}$ oxidase activation. Contribution to alterations of vasomotor tone. J Clin Invest 97:1916-1923

8. Pendergrass KD, Pirro NT, Westwood BM, Ferrario CM, Brosnihan KB, Chappell MC (2008) Sex differences in circulating and renal angiotensins of hypertensive mRen(2). Lewis but not normotensive Lewis rats. Am J Physiol Heart Circ Physiol 295:H10-H20

9. SANTOS R A S, OUDIT G Y, VERANO-BRAGA T, CANTA G, STECKELINGS U M BM (2019) The reninangiotensin system: going beyond the classical paradigms. Am J Physiol Hear Circ Physiol 1:316:H958-H970

10. OCARANZA M P, RIQUELME J A, GARCÍA L, JALIL J E, CHIONG M (2020) SANTOS R A. S LS. Counterregulatory renin-angiotensin system in cardiovascular disease. Nat Rev Cardiol 17:116-129

11. Hein S, Arnon E, Kostin S, Schönburg M, Elsässer A, Polyakova V et al (2003) Progression from compensated hypertrophy to failure in the pressure-overloaded human heart: structural deterioration and compensatory mechanisms. Circulation 107:984-991

12. Woodiwiss AJ, Tsotetsi OJ, Sprott S, Lancaster EJ, Mela T, Chung ES et al (2001) Reduction in myocardial collagen cross-linking parallels left ventricular dilatation in rat models of systolic chamber dysfunction. Circulation 103:155-160

13. Kotchen JM, McKean HE, Kotchen TA. Blood pressure trends with aging. Hypertens (Dallas, Tex 1979). 1982;4:III128-34

14. Regitz-Zagrosek V, Oertelt-Prigione S, Seeland U, Hetzer R (2010) Sex and gender differences in myocardial hypertrophy and heart failure. Circ J 74:1265-1273 
15. Bachmann H, Horacek U, Leowsky M, Hirche H (1987) [Blood pressure in children and adolescents aged 4 to 18. Correlation of blood pressure values with age, sex, body height, body weight and skinfold thickness (Essen Blood Pressure Study)]. Monatsschr Kinderheilkd 135:128-134

16. Harshfield GA, Alpert BS, Pulliam DA, Somes GW, Wilson DK (1994) Ambulatory blood pressure recordings in children and adolescents. Pediatrics 94:180-184

17. Dalpiaz PLM, Lamas AZ, Caliman IF, Ribeiro RF, Abreu GR, Moyses MR et al (2015) Sex Hormones Promote Opposite Effects on ACE and ACE2 Activity, Hypertrophy and Cardiac Contractility in Spontaneously Hypertensive Rats. PLoS One 10:e0127515

18. Sica DA, Gehr TWB (1999) Current Concepts of Pharmacotherapy in Hypertension - ACE Inhibitors: Current Considerations in Their Use. J Clin Hypertens (Greenwich) 1:218-230

19. Mersmann J, Tran N, Zacharowski PA, Grotemeyer D, Zacharowski K (2008) Rosiglitazone is cardioprotective in a murine model of myocardial I/R. Shock 30:64-68

20. Ikejima H, Imanishi T, Tsujioka H, Kuroi A, Kobayashi K, Shiomi M et al (2008) Effects of telmisartan, a unique angiotensin receptor blocker with selective peroxisome proliferator-activated receptorgamma-modulating activity, on nitric oxide bioavailability and atherosclerotic change. J Hypertens 26:964-972

21. Goyal S, Arora S, Bhatt TK, Das P, Sharma A, Kumari S et al (2010) Modulation of PPAR-gamma by telmisartan protects the heart against myocardial infarction in experimental diabetes. Chem Biol Interact 185:271-280

22. Vassallo DV, de Carvalho AP (1979) Influence of temperature on isometric contractions of rabbit atria during inotropic interventions. Acta Physiol Lat Am 29:131-135

23. Vassallo DV, Lebarch EC, Moreira CM, Wiggers GA, Stefanon I (2008) Lead reduces tension development and the myosin ATPase activity of the rat right ventricular myocardium. Brazilian J Med Biol Res $=$ Rev Bras Pesqui medicas e Biol 41:789-795

24. Vassallo DV, Mill JG (1988) Mechanical behavior of rest contractions in cardiac muscle. Acta Physiol Pharmacol Latinoam [Internet]. [cited 2018 Oct 22];38:87-97. Available from:

http://www.ncbi.nlm.nih.gov/pubmed/3201999

25. Leite CM, Vassallo DV, Mill JG (1991) Post-rest contractions of amphibian cardiac muscle. Brazilian $\mathrm{J}$ Med Biol Res $=$ Rev Bras Pesqui medicas e Biol 24:843-846

26. Mill JG, Vassallo DV, Leite CM (1992) Mechanisms underlying the genesis of post-rest contractions in cardiac muscle. Brazilian J Med Biol Res = Rev Bras Pesqui medicas e Biol 25:399-408

27. Ayaz O, Howlett SE (2015) Testosterone modulates cardiac contraction and calcium homeostasis: cellular and molecular mechanisms. Biol Sex Differ 6:9

28. Best CJM, Gillespie JW, Yi Y, Chandramouli GVR, Perlmutter MA, Gathright Y et al (2005) Molecular Alterations in Primary Prostate Cancer after Androgen Ablation Therapy. Clin Cancer Res 11:68236834

29. Tam NNC, Gao Y, Leung Y-K, Ho S-M (2003) Androgenic regulation of oxidative stress in the rat prostate: involvement of $\mathrm{NAD}(\mathrm{P}) \mathrm{H}$ oxidases and antioxidant defense machinery during prostatic 
involution and regrowth. Am J Pathol 163:2513-2522

30. Vutthasathien P, Wattanapermpool J (2015) Regular exercise improves cardiac contractile activation by modulating MHC isoforms and SERCA activity in orchidectomized rats. J Appl Physiol 119:831839

31. Witayavanitkul N, Woranush W, Bupha-Intr T, Wattanapermpool J (2013) Testosterone regulates cardiac contractile activation by modulating SERCA but not NCX activity. Am J Physiol Heart Circ Physiol 304:H465-H472

32. Tsang S, Wong SSC, Wu S, Kravtsov GM, Wong T-M (2009) Testosterone-augmented contractile responses to alpha1- and beta1-adrenoceptor stimulation are associated with increased activities of RyR, SERCA, and NCX in the heart. Am J Physiol Cell Physiol 296:C766-C782

33. Chen YF, Naftilan AJ, Oparil S. Androgen-dependent angiotensinogen and renin messenger RNA expression in hypertensive rats. Hypertens (Dallas, Tex 1979). 1992;19:456-63

34. Benson SC, Pershadsingh $\mathrm{HA}, \mathrm{Ho} \mathrm{Cl}$ et al (2004) Identification of telmisartan as a unique angiotensin II receptor antagonist with selective PPAR-gamma-modulating activity. Hypertension 43:993-1002

35. Cadeddu C, Piras A, Mantovani G, Deidda M, Dessì M, Madeddu C et al. Protective effects of the angiotensin II receptor blocker telmisartan on epirubicin-induced inflammation, oxidative stress, and early ventricular impairment. Am Heart J. 2010;160:487.e1-487.e7

36. Sharma AK, Kanawat DS, Mishra A, Dhakad PK, Sharma P, Srivastava V et al (2014) Dual therapy of vildagliptin and telmisartan on diabetic nephropathy in experimentally induced type 2 diabetes mellitus rats. J Renin Angiotensin Aldosterone Syst 15:410-418

37. Verdecchia P, Angeli F, Gentile G, Mazzotta G, Reboldi G (2011) Telmisartan for the reduction of cardiovascular morbidity and mortality. Expert Rev Clin Pharmacol 4:151-161

38. Derosa G, Ragonesi PD, Mugellini A, Ciccarelli L, Fogari R (2004) Effects of telmisartan compared with eprosartan on blood pressure control, glucose metabolism and lipid profile in hypertensive, type 2 diabetic patients: a randomized, double-blind, placebo-controlled 12-month study. Hypertens Res 27:457-464

39. Bers DM. coupling. 2002;415

\section{Figures}


A

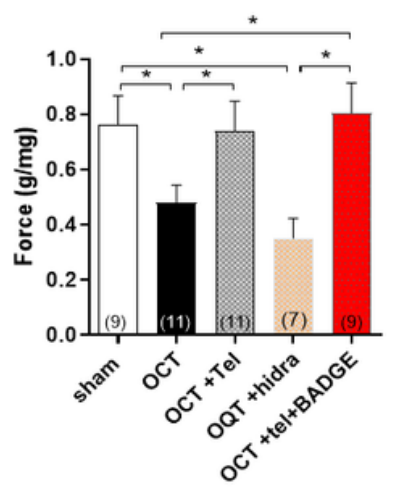

B

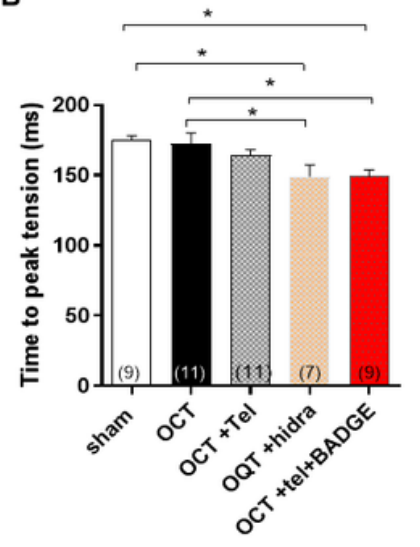

C

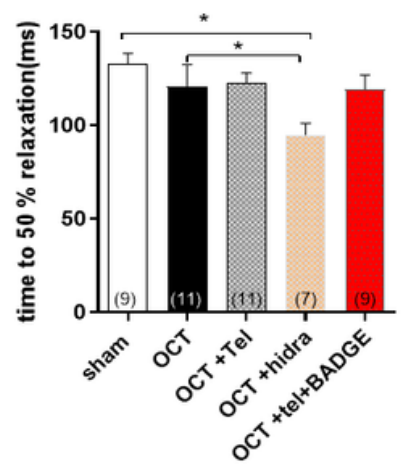

D

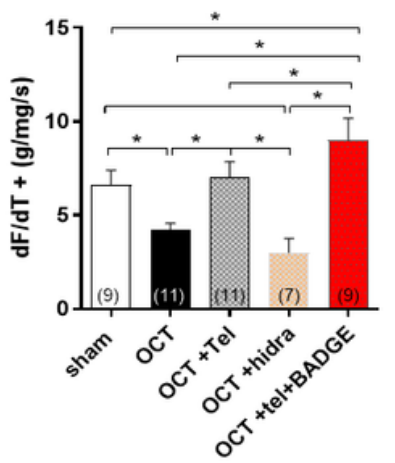

E

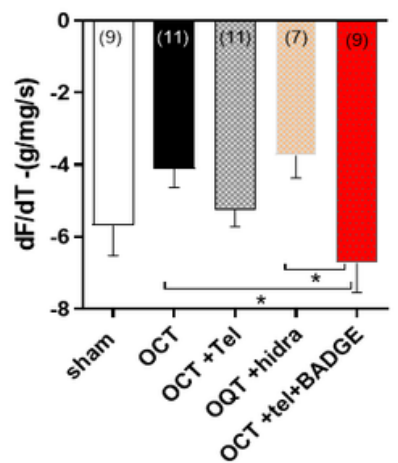

\section{Figure 1}

Developed isometric force, first derived from force development and temporal parameters of contraction of the left ventricular papillary muscles of rats of all experimental groups (sham- group control, OCTorchiectomized, OCT+Tel-orchiectomized treated with telmisartan, OCT+hidra-orchiectomized treated with hydralazine, OCT+Tel+BADGE- orchiectomized treated with temisartan and PPARY inhibitor). (A) Isometric force, (B) time to peak tension and (C) time to $50 \%$ relaxation, (D) maximum positive derivative and $(\mathrm{E})$ maximum negative force derivative $(\mathrm{dF} / \mathrm{dt}+$ and $\mathrm{dF} / \mathrm{dt}$ - respectively). The numbers in parentheses correspond to the number of papillaries studied. Data are expressed as mean \pm SEM. * $P$ $<0.05$, oneway ANOVA followed by Fischer post hoc test for multiple comparisons. 


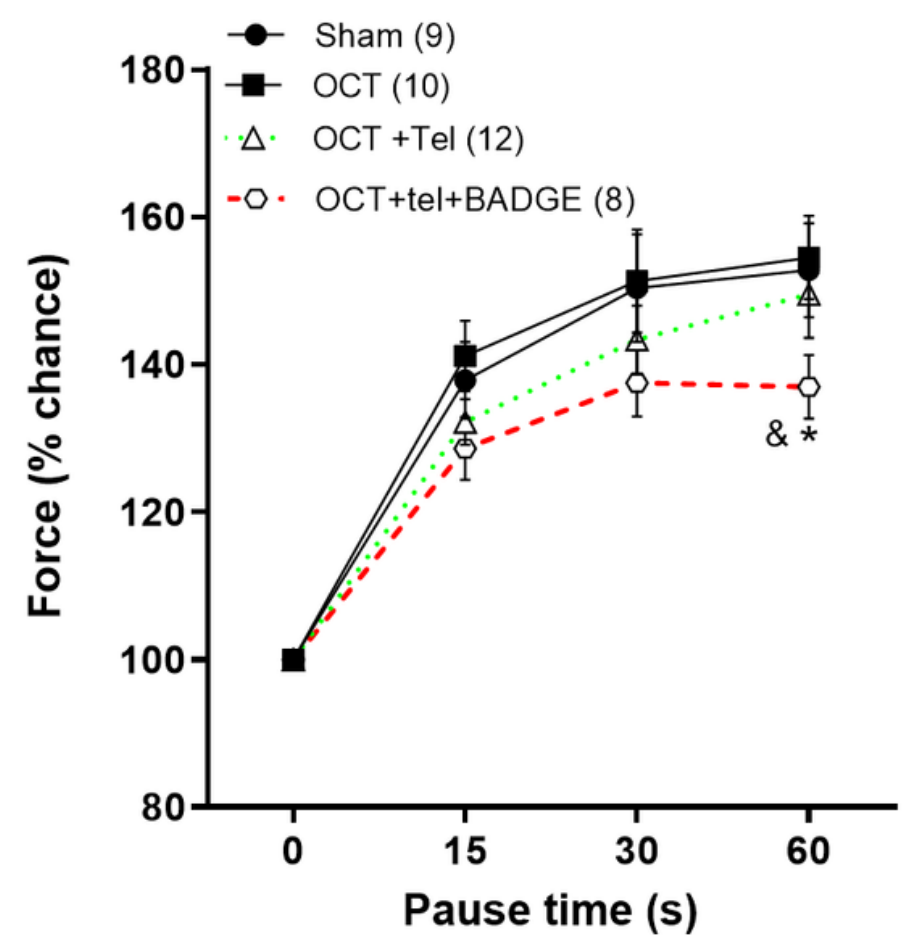

Figure 2

Evaluation, in vitro, of the relative potentiation after pauses of 15, 30 and 60 seconds of LV papillary muscles of Sham rats, orchiectomized (OCT), orchiectomized treated with telmisartan (OCT + Tel), orchiectomized treated with telmizartan and BADGE (OCT + Tel + BADGE). The numbers in parentheses correspond to the number of Papillary muscles studied. Data are expressed as mean $\pm S E M$. * $P<0.05$ vs Sham; \& $\mathrm{P}<0.05$ vs OQT: Two-way ANOVA followed by Fischer post-hoc test for multiple comparisons. 

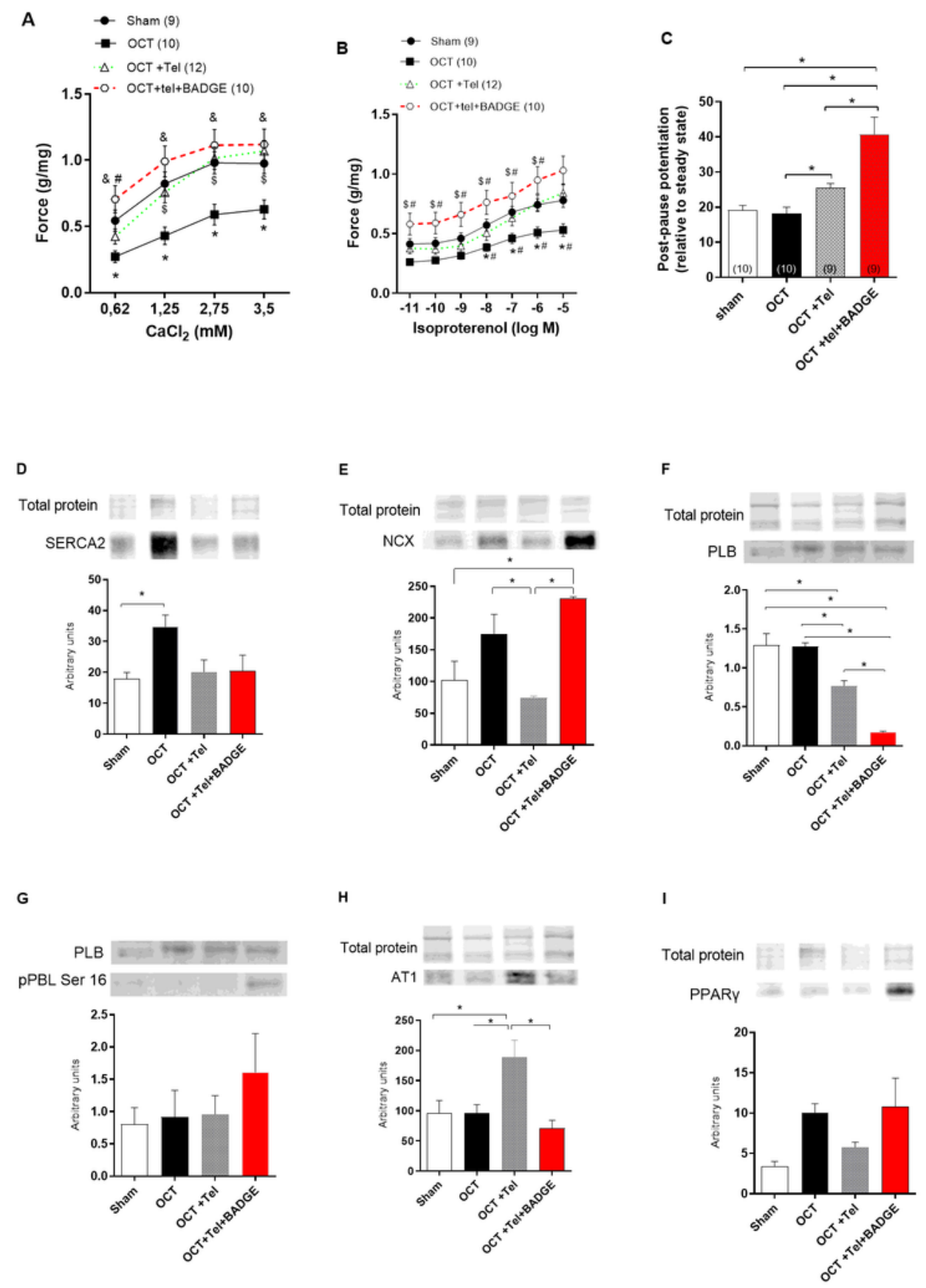

\section{Figure 3}

Contractile response to different experimental protocols and densitometric analysis of Western blots of Sham, orchiectomized (OCT), telmisartan treated orchiectomized (OCT + Tel), telmizartan and BADGE orchiectomized treatments (OCT + Tel + BADGE). A) Strength developed during increments of Ca2+ concentration, (B) Inotropic responses of cardiac muscle to isoproterenol, (C) Contraction dependent on transarcolomal calcium influx, (D) SERCA2 (6), (E) NCX (6 ), (F) PBL (6), (G) pPBLSer16 (6), (H) AT1 (6), (I) 
PPARg (6). The numbers in parentheses correspond to the number of papillaries studied. Data are expressed as mean \pm SEM. * $P<0.05$ vs Sham; $\$ P<0.05$ vs OCT, \# $P<0.05$ vs OCT + Tel, \& $P<0.05$ vs OQT OCT BADGE.

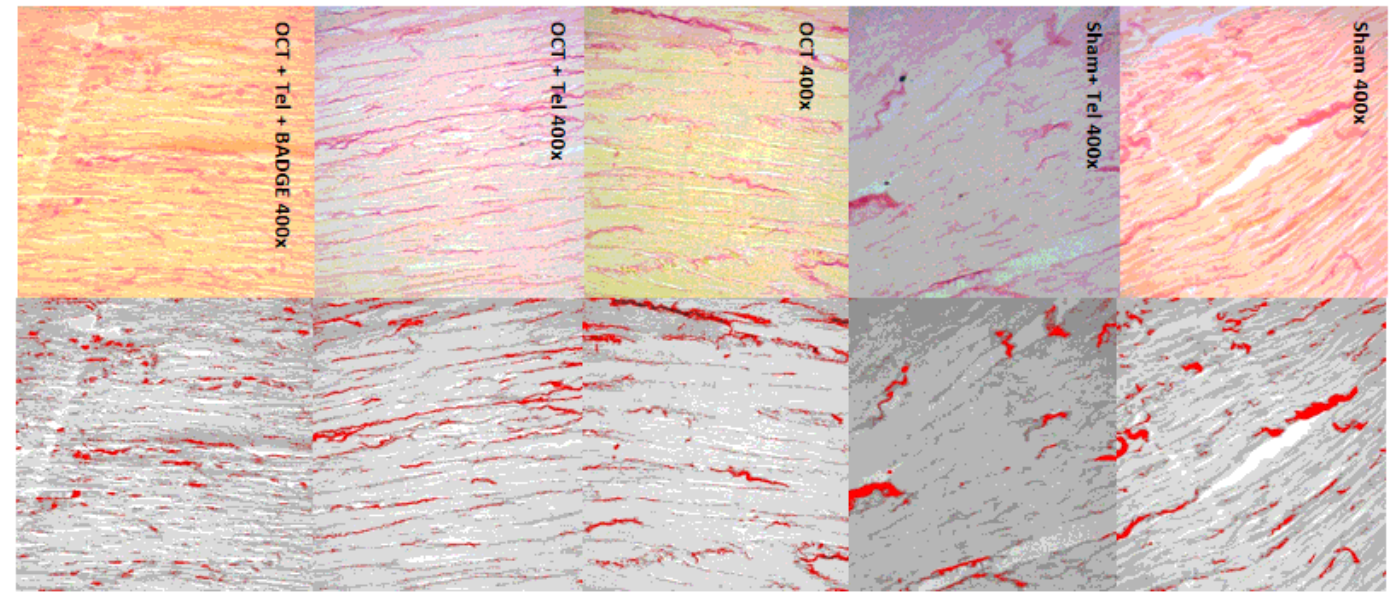

A

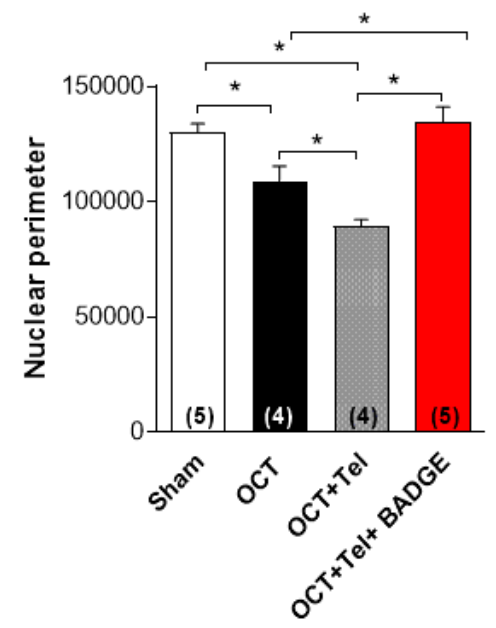

B

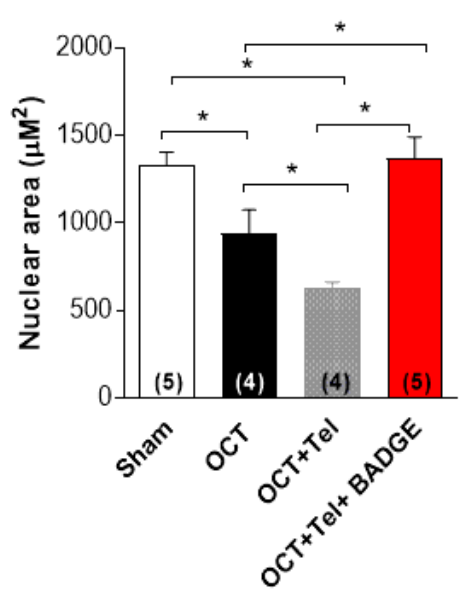

C

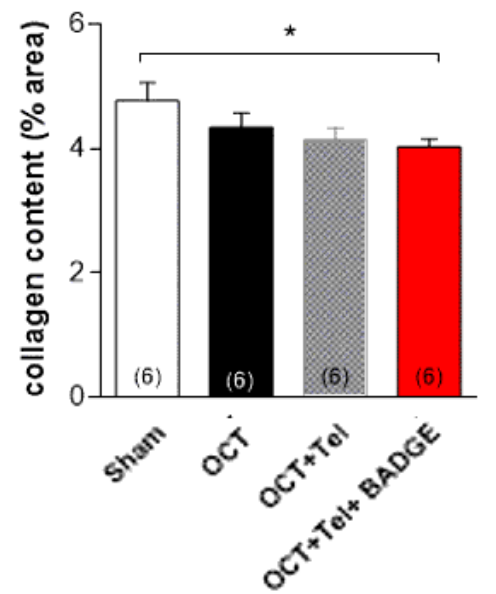

Figure 4

Nuclear size and collagen deposition indicators in the left ventricle. (A) Nuclear perimeter, (B) Nuclear area, (C) Collagen content of of Sham, orchiectomized (OCT), telmisartan treated orchiectomized (OCT + Tel), telmizartan and BADGE orchiectomized treatments (OCT + Tel + BADGE). The numbers in 
parentheses correspond to the number of papillaries studied. Data are expressed as mean \pm SEM. * $P$ $<0.05$, one-way ANOVA followed by Fischer post-hoc test for multiple comparisons

\section{Supplementary Files}

This is a list of supplementary files associated with this preprint. Click to download.

- Graphicalabstract2021.jpg 